\title{
THE QUADRATIC LAW OF RECIPROCITY AND THE THEORY OF GALOIS FIELDS
}

\author{
by HANS J. ZASSENHAUS \\ (Received 18th November, 1950)
}

The theory of quadratic congruences modulo an integer is dominated by the Quadratic Law of Reciprocity (see §1), which makes it possible to decide in a very short time whether a quadratic congruence

$$
x^{2} \equiv a(m)
$$

is solvable or not. The law was first proved by Gauss.* It took him over a year to obtain his first proof, which depends on a tedious lemma in elementary number theory. He subsequently obtained seven further proofs, and today more than fifty proofs are known, most of them based on the ideas of Gauss. The object of the present paper is to present a proof which is a modernised version of Gauss's seventh proof, applying the ideas of that proof to a finite set of objects, the elements of a finite or Galois field.

The first section of this paper gives a group-theoretic treatment of the elementary properties of Legendre's symbol. In the second section we introduce Gaussian sums in finite fields. A comparison of two different expressions for these yields the quadratic law of reciprocities.

We conclude this introduction by giving a summary of the properties of Galois fields required in the course of the proof of the quadratic law of reciprocity. $N-1$.

1. The multiplicative group of a Galois field of $N$ elements is a cyclical group of order

2. A Galois field has for its characteristic a prime number $p$, and therefore for its prime field a field isomorphic with $F_{p}$. the field of residue classes of the integers modulo $p$. We shall denote any field isomorphic with this field by $F_{p}$. The number of elements in any Galois field of characteristic $p$ is of the form $p^{r}$ where $r$ is a positive integer. Conversely, corresponding to any prime number $p$ and any positive integer $r$, there is a Galois field of characteristic $p$ and with $p^{r}$ elements. This Galois field, which is denoted by $G F\left(p^{r}\right)$, is uniquely determined up to isomorphism as a minimal splitting field of the polynomial $t^{p^{r}}-t$ over the field $\boldsymbol{F}_{p}$.

3. The prime field $F_{p}$ of $G F\left(p^{r}\right)$ consists of the set of $p$ elements of $G F\left(p^{r}\right)$ which satisfy the equation

$$
x^{p}=x .
$$

4. The correspondence $\sigma$ defined by

is an automorphism of $G F\left(p^{r}\right)$.

$$
x \rightarrow x^{p}=x^{\sigma}, \quad x \in G F\left(p^{r}\right),
$$

The proofs of these properties of Galois fields will be found in books on Modern Algebra or in the introduction to another paper in this number of these proceedings. (A Grouptheoretic Proof of a Theorem of Maclagan-Wedderburn, pp. 53-63).

$\S 1$. The Legendre Symbol.

The Legendre symbol arises in connection with the problem of solving a quadratic congruence

$$
a x^{2}+b x+c \equiv 0(m),
$$

* Gauss, Disquisitiones Arithmeticae. Proof 1 : Vol. I, p. 135 ; proof 7 : Vol. II, p. 234. 
i.e., of finding an integer $x$ which satisfies this relation. It can be shown that this problem can be reduced to that of solving a quadratic congruence of the form

where $p$ is an odd prime.

$$
x^{2} \equiv d(p),
$$

It is much more important to establish a means of determining whether (1) has a solution than to find its set of solutions if it has any. To deal with the former problem, Legendre introduced a symbol $\left(\frac{d}{p}\right)$, defined in the following way :

$$
\begin{aligned}
& \left(\frac{d}{p}\right)=1 \text { if } d \equiv \equiv 0(p), \text { but }(1) \text { has a solution; } \\
& \left(\frac{d}{p}\right)=-1 \text { if }(1) \text { has no solution; } \\
& \left(\frac{d}{p}\right)=0 \text { if } d \equiv 0(p) .
\end{aligned}
$$

This Legendre symbol is defined for every integer $d$ in the numerator and for every prime greater than 2 in the denominator. Its value, for a given $d$ and $p$, is either $1,-1$ or 0 . Obviously, from (1), its value is unaltered when $d$ is replaced by any integer congruent to $d$ modulo $p$. Thus its value depends only on the residue class $R_{d}$ of integers modulo $p$ to which $d$ belongs. Thus we can define uniquely, by

$$
\left(\frac{R_{d}}{d}\right)=\left(\frac{d}{p}\right)
$$

the Legendre symbol of each element of $F_{p}$, the field of residue classes of the integers modulo $p$. Further, if we observe that, if any integer $x$ satisfies (1), so do all the integers congruent to $x$ modulo $p$, we see that the problem of solving the congruence (1) in the domain of all integers is equivalent to that of solving the equation

$$
x^{2}=\alpha,
$$

where $\alpha$ is an element of $F_{p}$, in the field $F_{p}$. In consequence of this, the definition of the Legendre symbol $\left(\frac{\alpha}{p}\right)$, where $\alpha$ is an element of $F_{p}$, can be restated thus :

$\left(\frac{\alpha}{p}\right)=1$ if $\alpha$ is not 0, the zero element of $F_{p}$, and is the square of an element of $F_{p} ;$

$\left(\frac{\alpha}{p}\right)=-1$ if $\alpha$ is not the square of any element of $F_{p}$;

$\left(\frac{0}{p}\right)=0$.

Since $p$ is an odd prime, the elements $1,-1$ and 0 of $F_{p}$ are distinct, so the values of the Legendre symbol, hitherto considered as the integers $1,-1$ and 0 , may equally well be considered as the elements $1,-1$ and 0 of $F_{p}$. This interpretation is frequently more convenient, for it often allows congruences to be replaced by equations. This is the case in the following important theorem of Euler.

$$
\left(\frac{\alpha}{p}\right)=\alpha^{\frac{1}{2}(p-1)}
$$

Proof : If $\alpha=0$, both sides of (3) are zero, and therefore (3) is true.

To deal with the case in which $\alpha \neq 0$, consider the correspondence

$$
\xi \rightarrow \xi^{2}
$$


where $\xi$ is a non-zero element of $F_{p}$. This correspondence is an operator of the multiplicative group of $F_{p}$, i.e., it is a homomorphism of this group onto a subgroup ; for $(\xi \eta)^{2}=\xi^{2} \eta^{2}$. The kernel of this homomorphism is the set of elements of $F_{p}$ for which $\xi^{2}=1$, i.e., since $F_{p}$ is a field, it is the two elements +1 and -1 of $F_{p}$. By the fundamental theorem on homomorphisms, it follows that the image of $F_{p}$ under the homomorphism, i.e., the set of elements of $F_{p}$ which are non-zero and the squares of elements of $F_{p}$, forms a multiplicative group $S_{p}$ isomorphic with the factor group of the multiplicative group of $F_{\mathfrak{p}}$ over the normal divisor consisting of the pair of elements \pm 1 . The order of $S_{p}$ is therefore half that of the multiplicative group of $F_{p}$, i.e.,

$$
S_{p}: 1=\frac{1}{2}(p-1)
$$

Now, for each non-zero element of $F_{p}$, we have, by Fermat's theorem,

$$
\text { i.e., } \quad \begin{gathered}
\xi^{p-1}=1, \\
\left(\xi^{2}\right)^{\frac{1}{(p-1)}}=1 .
\end{gathered}
$$

Hence (3) is true for all elements of $S_{p}$. Further, since the equation $\xi^{\xi^{(p-1)}}=1$ cannot be satisfied

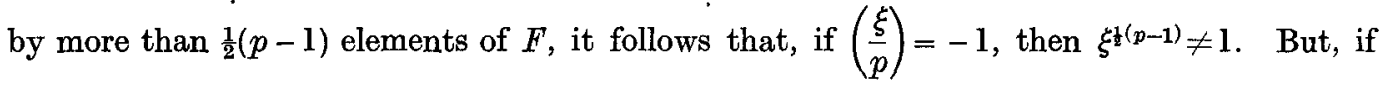
$\xi \neq 0,\left\{\xi^{\frac{1}{2}(p-1)}\right\}^{2}=1$ and therefore $\xi^{\frac{1}{2}(p-1)}= \pm 1$. Hence,

$$
\text { if } \quad\left(\frac{\xi}{p}\right)=-1 \text {, then } \xi^{\frac{1}{2}(p-1)}=-1 \text {. }
$$

The formula (3) is thus established for all elements $\alpha$ of $F_{p}$.

From (3) it follows that

$$
\left(\frac{\alpha \beta}{p}\right)=\left(\frac{\alpha}{p}\right)\left(\frac{\beta}{p}\right)
$$

i.e., that, in $F_{p}$,

$$
0 . \alpha=\alpha \cdot 0=0 \text {, }
$$

where 0 is the zero element of $F_{p}$; while, for non-vanishing products,

$$
\text { square } \times \text { square }=\text { square, }
$$

square $\times$ non-square $=$ non-square $\times$ square $=$ non-square, non-square $\times$ non-square $=$ square.

It follows also that

$$
\left(\frac{-1}{p}\right)=(-1)^{\frac{1}{2}(p-1)}
$$

i.e., that

$$
\begin{aligned}
& \left(\frac{-1}{p}\right)=1 \text { if } p \equiv 1(4), \\
& \left(\frac{-1}{p}\right)=-1 \text { if } p \equiv 3(4) .
\end{aligned}
$$

The quadratic law of reciprocity and its complements $I$ and II state the following properties of the Legendre symbols.

If $p$ and $q$ are different odd primes, then

$$
\left(\frac{p}{q}\right)=\left(\frac{q}{p}\right) \text { if either } p \equiv 1(4) \text { or } q \equiv 1(4)
$$

and

$$
\left(\frac{p}{q}\right)=-\left(\frac{q}{p}\right) \text { if } p \equiv q \equiv 3(4) \text {. }
$$


Complement I : $\quad\left(\frac{-1}{q}\right)=1$ if $q \equiv 1(4)$,

$$
\left(\frac{-1}{q}\right)=-1 \text { if } q \equiv 3(4) \text {. }
$$

Complement II : $\quad\left(\frac{2}{q}\right)=1$ if $q \equiv \pm 1(8)$,

$$
\left(\frac{2}{q}\right)=-1 \text { if } q \equiv \pm 3(8) \text {. }
$$

These statements may be collected into the following more compact forms :

The Quadratic Law of Reciprocity :

$$
\left(\frac{p}{q}\right)\left(\frac{q}{p}\right)=(-1)^{\frac{1}{2}(p-1) \cdot \frac{1}{2}(q-1)}
$$

Complement I :

$$
\left(\frac{-1}{q}\right)=(-1)^{\frac{1}{2}(q-1)} \text {. }
$$

Complement II :

$$
\left(\frac{2}{q}\right)=(-1)^{\frac{1}{8}\left(q^{2}-1\right)}
$$

The relation (6) is obviously equivalent to the Quadratic Law of Reciprocity itself, the relation (7) is the same as (5) and has therefore been proved already. To prove the relation (8) we express $q$ in the form $8 l+(2 k+1)$, where $k$ and $l$ are integers, and observe that

$$
\begin{aligned}
\frac{1}{8}\left(q^{2}-1\right) & =8 l^{2}+2 l(2 k+1)+\frac{1}{2} k(k+1) \\
& \equiv \frac{1}{2} k(k+1) \quad(\bmod 2) \\
& \equiv \frac{1}{8}\left\{(2 k+1)^{2}-1\right\} \quad(\bmod 2) .
\end{aligned}
$$

Thus the value of $(-1)^{\frac{1}{3}\left(q^{2}-1\right)}$ depends only on the residue class of $q$ modulo 8 , and the value is 1 if $q \equiv \pm 1$ (8) and -1 if $q \equiv \pm 3$ (8).

If we introduce the symbol

we may write (6) in the form

$$
\epsilon=(-1)^{\frac{1}{2}(q-1)}=\left(\frac{-1}{q}\right),
$$

$$
\left(\frac{p}{q}\right)\left(\frac{q}{p}\right)=\epsilon^{\frac{1}{2}(p-1)}=\left(\frac{\epsilon}{p}\right)
$$

and hence, after multiplication on both sides by $\left(\frac{q}{p}\right)$, in the form

$$
\left(\frac{p}{q}\right)=\left(\frac{\epsilon q}{p}\right)
$$

§2. Proof of the Quadratic Law of Reciprocity and its Second Complement.

Let $p$ and $q$ be two different positive prime numbers. Let $f$ be the order of $p$ modulo $q$, i.e., let $f$ be the positive integer for which $q$ is a factor of $p^{f}-1$ but is not a factor of $p^{v}-1$, where $0<v<f$. Then the multiplicative group of the Galois field $G F\left(p^{f}\right)$, being cyclical of order $p^{f}-1$, contains an element $\zeta$ of order $q$ and therefore such that

Since

$$
\zeta^{a}=1 \text { but } \zeta \neq 1
$$

and $\zeta \neq 1$, it follows that

$$
\zeta^{a}-1=(\zeta-1)\left(\zeta^{q-1}+\zeta^{a-2}+\ldots+1\right)
$$

$$
\sum_{i=0}^{q-1} \zeta^{i}=0
$$


Since, if $a$ and $b$ are two integers, $\zeta^{a}=\zeta^{b}$ if and only if $a \equiv b(q)$, it is possible to define $\zeta^{\alpha}$, where $\alpha$ is any residue class of the integers modulo $q$, thus :

$$
\zeta^{\alpha}=\zeta^{a},
$$

where $a$ is any integer in the residue class $\alpha$. As a result of this definition,

$$
\zeta^{\alpha} \zeta^{\beta}=\zeta^{\alpha+\beta},
$$

where $\alpha$ and $\beta$ are any residue classes of the integers modulo $q$, i.e., any elements of $F_{q}$.

We now define, for each prime number $p$ and each element $\alpha$ of $F_{q}$, the Gaussian sum $G(\alpha, p)$, thus :

$$
G(\alpha, p)=\Sigma \zeta^{\alpha \xi},
$$

where summation is over all elements $\xi$ of $F_{\alpha}$ for which $\left(\frac{\xi}{q}\right)=1$. The Gaussian sum is an element of $G F\left(p^{f}\right)$. If $\alpha=0$, the zero element of $F_{\alpha}$, each of the $\frac{1}{2}(q-1)$ terms on the right side of (2) is equal to 1 , the unity element of $G F\left(p^{f}\right)$ and therefore $G(0, p)$ is equal to $\frac{1}{2}(q-1)$ times the unity element of $G F\left(p^{f}\right)$. If $\alpha$ is not the zero element of $F_{q}, G(\alpha, p)$ is equal to $\Sigma \zeta^{\beta}$, summation being over all elements $\beta$ of $F_{q}$ which have the same Legendre symbol as $\alpha$. It follows that the Gaussian sum depends only on $p, q$ and the Legendre symbol $\left(\frac{\alpha}{q}\right)$ of $\alpha$. Suppressing $p$, we may therefore introduce the notation

$$
G\left(\frac{\alpha}{q}\right)=G(\alpha, p)
$$

We note that the suffix $\left(\frac{\alpha}{q}\right)$ can only take the values $1,-1$ and 0 . We now find the values of $G_{1}, G_{-1}$ and $G_{0}$.

As we have just shown, $G_{0}$, i.e., $G(0, p)$, is equal to $\frac{1}{2}(q-1)$ times the unity element of $G F\left(p^{f}\right)$ and therefore of its subfield $F_{p}$. Hence

$$
G_{0}=\frac{1}{2}(q-1) \text { times the unity element of } F_{p} \text {. }
$$

To evaluate $G_{1}$ and $G_{-1}$, we note that

$$
\begin{aligned}
G_{1}+G_{-1} & =\underset{\left(\frac{\alpha}{q}\right)=1}{\sum} \zeta^{\alpha}+\underset{\left(\frac{\beta}{q}\right)=-1}{\sum \zeta^{\beta}} \\
& =\underset{0 \neq \gamma \in F_{q}}{\sum} \zeta^{\gamma}=-1, \ldots
\end{aligned}
$$

by (1), where 1 is the unity of $G F\left(p^{f}\right)$, i.e., of its subfield $F_{p}$; and that

$$
\begin{aligned}
G_{1} G_{-1} & =\sum \zeta^{\alpha} \sum \zeta^{\beta} \\
& =\sum_{\left(\frac{\alpha}{q}\right)=1}\left(\frac{\beta}{q}\right)=-1 \\
\left(\frac{\alpha}{q}\right)=-\left(\frac{\beta}{q}\right)=1 & \zeta^{\alpha+\beta}=\sum_{\gamma \in F_{q}} n_{\gamma} \zeta^{\gamma},
\end{aligned}
$$

where, for each $\gamma$ in $F_{q}, n_{\gamma}$ is the number of solutions $(\alpha, \beta)$ of the equation

$$
\gamma=\alpha+\beta, \text { with }\left(\frac{\alpha}{q}\right)=-\left(\frac{\beta}{q}\right)=1 .
$$

Now if $\xi$ is any element of $F_{q}$ with $\left(\frac{\xi}{q}\right)=1$, there is a one-one correspondence between the solutions of (5) and the solutions $\alpha^{\prime}=\alpha \dot{\xi}, \beta^{\prime}=\beta \xi$ of the equations

$$
\gamma \xi=\alpha^{\prime}+\beta^{\prime}, \text { with }\left(\frac{\alpha^{\prime}}{q}\right)=-\left(\frac{\beta^{\prime}}{q}\right)=1
$$


There is also, for each $\delta$ for which $\left(\frac{\delta}{q}\right)=-\left(\frac{\gamma}{q}\right)$, a one-one correspondence between the solutions of (5) and the solutions $\alpha^{\prime \prime}=\beta \gamma^{-1} \delta, \beta^{\prime \prime}=\alpha \gamma^{-1} \delta$ of the equation

$$
\delta=\alpha^{\prime \prime}+\beta^{\prime \prime}, \text { with }\left(\frac{\alpha^{\prime \prime}}{q}\right)=-\left(\frac{\beta^{\prime \prime}}{q}\right)=1
$$

Since, by suitable choices of $\xi$ and $\delta, \gamma \xi$ and $\delta$ can be made to be any elements of $F_{q}$ with Legendre symbols 1 and -1 respectively, it follows that $n_{\gamma}$ has the same value for all non-zero elements $\gamma$ of $F_{\alpha}$; let this value be $n$.

If $n_{0}>0$, then the equation

$$
0=\alpha+\beta, \text { with }\left(\frac{\alpha}{q}\right)=-\left(\frac{\beta}{q}\right)=-1,
$$

has at least one solution. For any such solutions $\beta=-\alpha$, i.e., $-1=\beta \alpha^{-1}$, and therefore

$$
\left(\frac{-1}{q}\right)=\left(\frac{\beta}{q}\right)\left(\frac{\alpha^{-1}}{q}\right)=\left(\frac{\beta}{q}\right)\left(\frac{\alpha}{q}\right)^{-1}=-1 \text {. }
$$

Conversely, if $\left(\frac{-1}{q}\right)=-1$ and $\alpha$ is any element of $F_{a}$, and if $\beta=-\alpha$, then $\left(\frac{\beta}{q}\right)=-1$; there is thus one solution of $(8)$ for every $\alpha$ for which $\left(\frac{\alpha}{q}\right)=1$. Hence

or, using the symbol

$$
n_{0}=\left\{\begin{array}{cl}
0 & \text { if }\left(\frac{-1}{q}\right)=1, \\
\frac{1}{2}(q-1) & \text { if }\left(\frac{-1}{q}\right)=-1 ;
\end{array}\right.
$$

introduced earlier,

$$
\epsilon=\left(\frac{-1}{q}\right)=(-1)^{\frac{1}{2}(q-1)},
$$

Since, in the sum

$$
n_{0}=\frac{1}{2}(1-\epsilon) \cdot \frac{1}{2}(q-1)
$$

$$
\begin{gathered}
\left.\Sigma \sum_{q}^{\alpha}\right)=-\left(\frac{\beta}{q}\right)=1 \\
\zeta^{\alpha+\beta}
\end{gathered}
$$

the total number of terms is $\left\{\frac{1}{2}(q-1)\right\}^{2}$, we have, from (4),

hence, using (9), we have

$$
n_{0}+(q-1) n=\left\{\frac{1}{2}(q-1)\right\}^{2}
$$

But, by (4),

$$
n=\frac{1}{4}(q+\epsilon-2) \text {. }
$$

$$
G_{1} G_{-1}=n_{0} \zeta^{0}+n \sum_{0 \neq \gamma \in F_{Q}} \zeta^{\nu}
$$

Hence,

$$
=\left(n_{0}-n\right) \text { times the unity element of the } F_{p} \text { in } G F\left(p^{f}\right) \text {. }
$$

$$
G_{1} G_{-1}=\frac{1}{4}(1-\epsilon q) \text { times the unity of the } F_{p} \text { in } G F\left(p^{f}\right) .
$$

From (3) and (10) it follows that $G_{1}$ and $G_{-1}$ are the elements of $G F\left(p^{f}\right)$ which satisfy the quadratic equation

$$
x^{2}+x+\frac{1}{4}(1-\epsilon q)=0
$$

where it is to be understood that $\frac{1}{4}(1-\epsilon q)$ means this integer multiplied by the unity element of the $F_{p}$ in $G F\left(p^{f}\right)$. 
If $p>2$, the usual method can be used to solve the quadratic equation, giving

$$
\left(x+\frac{1}{2}\right)^{2}=\frac{1}{4} \in q .
$$

Hence if a suitable solution in $G F\left(p^{f}\right)$ of $y^{2}=\epsilon q$ is denoted by $\sqrt{\epsilon q}$, we have

for $\delta=+1$ and -1 .

$$
G_{\delta}=-\frac{1}{2}+\frac{1}{2} \delta \sqrt{\epsilon q}
$$

Now, whether $p>2$ or not, the correspondence

$$
\eta \rightarrow \eta^{p}, \quad \eta \epsilon G F\left(p^{f}\right),
$$

is an automorphism of $G F\left(p^{f}\right)$. Applying this automorphism to $G(\alpha, p)$, we have

$$
\{G(\alpha, p)\}^{p}=\left(\begin{array}{c}
\Sigma \zeta^{\alpha \xi} \\
\left(\frac{\xi}{q}\right)=1
\end{array}\right)^{p}=\sum_{\left(\frac{\xi}{q}\right)=1}^{\Sigma} \zeta^{\alpha \xi p}=G(\alpha p, p)
$$

and therefore, using the other notation for the Gaussian sum and putting $\left(\frac{\alpha}{q}\right)=\delta$, where $\delta= \pm 1$, we have

$$
\left(G_{\delta}\right)^{p}=G\left(\frac{p}{q}\right) \delta
$$

since $\left(\frac{\alpha p}{q}\right)=\left(\frac{\alpha}{q}\right)\left(\frac{p}{q}\right)=\delta\left(\frac{p}{q}\right)$.

In the case in which $p>2$,

$$
\left(G_{\delta}\right)^{p}=\left(-\frac{1}{2}+\frac{1}{2} \delta \sqrt{\epsilon q}\right)^{p}=\left(-\frac{1}{2}\right)^{p}+\left(\frac{1}{2} \delta\right)^{p}(\sqrt{\epsilon q})^{p}=-\frac{1}{2}+\frac{1}{2} \delta(\sqrt{\epsilon q})^{p}
$$

since $-\frac{1}{2}$ and $\frac{1}{2} \delta$ lie in the subfield $F_{p}$ of $G F\left(p^{f}\right)$; and

It follows from (13) that

$$
G_{\left(\frac{p}{q}\right) \delta}=-\frac{1}{2}+\frac{1}{2}\left(\frac{p}{q}\right) \delta \sqrt{\epsilon q}
$$

$$
(\sqrt{\epsilon q})^{p}=\left(\frac{p}{q}\right) \delta \sqrt{\epsilon q}
$$

Thus, if $\left(\frac{p}{q}\right)=1$,

$$
(\sqrt{\epsilon q})^{p}=\sqrt{\epsilon q}
$$

hence $\sqrt{\epsilon q}$ is in $F_{p}$ and therefore $\left(\frac{\epsilon q}{p}\right)=1$. In this case, therefore, $\left(\frac{p}{q}\right)=\left(\frac{\epsilon q}{p}\right)$. If $\left(\frac{p}{q}\right)=-1$

$$
(\sqrt{\epsilon q})^{p}=-\sqrt{\epsilon q}
$$

hence $\sqrt{\epsilon q}$ is not in $F_{p}$ and therefore $\left(\frac{\epsilon q}{p}\right)=-1$. Thus, in this case also $\left(\frac{p}{q}\right)=\left(\frac{\epsilon q}{p}\right)$.

This completes the proof of the quadratic law of reciprocity itself.

There remains to be completed the proof of the second complement, dealing with the case in which $p=2$. In that case (13) reads

$$
\begin{aligned}
& \left(G_{\delta}\right)^{2}=G\left(\frac{2}{q}\right) \delta^{\prime} \\
& \left(G_{\delta}\right)^{2}= \begin{cases}G_{\delta} & \text { if }\left(\frac{2}{q}\right)=1 \\
G_{-\delta} & \text { if }\left(\frac{2}{q}\right)=-1 .\end{cases}
\end{aligned}
$$


We note that if $x$ is either element of the subfield $F_{2}$ of $G F\left(2^{f}\right)$, then $x^{2}+x=0$, and that therefore no other element of $G F\left(2^{f}\right)$ satisfies this equation. This has the following consequences.

If $\frac{1}{4}(1-\epsilon q) \equiv 0(2)$, then both elements of $F_{2}$ are roots of (11) and no other elements of $G F\left(2^{f}\right)$ are. Hence, for each $\delta, G_{\delta} \in F_{2}$ and therefore $G_{\delta}{ }^{2}=G_{\delta}$. Thus, by (14), $\left(\frac{2}{q}\right)=1$.

If $\frac{1}{4}(1-\epsilon q) \equiv \mathrm{I}(2)$, then neither element of $F_{2}$ is a root of (11), and therefore neither of the roots $G_{\delta}$ of (11) lies in $F_{2}$. Hence, for these roots $G_{\delta}, G_{\delta}^{2} \neq G_{\delta}$ (for the two elements of $F_{2}$ and therefore no other elements of $G F\left(2^{f}\right)$ satisfy the equation $\left.x^{2}=x\right)$. Hence $G_{\delta}^{2}=G_{-\delta}$ and therefore, by (14), $\left(\frac{2}{q}\right)=-1$.

Now if $\frac{1}{4}(1-\epsilon q) \equiv 0(2), 1-\epsilon q \equiv 0(8)$, i.e., $\epsilon q \equiv \mathbf{l}(8)$ and therefore $q \equiv \pm 1$ (8); while if $\frac{1}{4}(1-\epsilon q) \equiv 1(2), 1-\epsilon q \equiv 4(8)$, i.e., $\epsilon q \equiv-3(8)$ and therefore $q \equiv \pm 3(8)$. Hence, if

$$
q \equiv \pm 1 \cdot(8),\left(\frac{2}{q}\right)=1 ; \text { if } q \equiv \pm 3(8),\left(\frac{2}{q}\right)=-1
$$

This completes the proof of the second complement.

MaGILl UnIVERSITY,

Montreal, Canada. 